- Attitudes to binge drinking among adolescent males are difficult to change using traditional health promotion messages.

- This paper demonstrates that an orofacial trauma-based brief intervention can change

attitudes to binge drinking among adolescent males, but not drinking behaviour.

- An orofacial trauma-based brief intervention could be the first step of a larger intervention which may reduce binge drinking among adolescent males.

\title{
Facing up to binge drinking: reducing binge drinking in adolescent males
}

\author{
M. Dempster, ${ }^{1}$ G. Newell, ${ }^{2}$ G. Cowan ${ }^{3}$ and J. Marley ${ }^{4}$
}

\begin{abstract}
Objective To evaluate the effectiveness of an orofacial trauma-based brief intervention, designed to raise adolescent males' awareness about the immediate dangers of binge drinking.

Design Non-randomised controlled exploratory trial.

Setting Secondary level schools.

Materials \&t methods Pre, post and follow up validated questionnaires were used to assess a variety of descriptive data and changes in behaviour. Sixty Year 12 students were recruited in the pilot study and 182 in the definitive study.

Intervention A brief visual presentation containing salient information and anonymised photographs relating to orofacial injuries.

Main outcome measures Intention to binge drink.

Results The majority of participants obtained alcohol from off-licence or licenced premises. At the commencement of the study, 68\% of the participants were regular drinkers. Whilst there was no change in drinking behaviour, the intervention group reported that it was significantly more likely (compared to the control group) that they would reduce their drinking to less than binge levels.

Conclusion The intervention resulted in participants reporting a more negative attitude towards binge drinking and increased their intention to disengage from binge drinking.
\end{abstract}

\section{INTRODUCTION}

Binge drinking (drinking half the recommended maximum weekly consumption of alcohol in a single session) is associated with high levels of morbidity and mortality and is the commonest mode of alcohol consumption by adolescents. ${ }^{1}$ There is widespread public and governmental concern about the level of alcohol consumption among adolescents, ${ }^{1,2}$ particularly in light of the recent extension of drinking hours. ${ }^{3}$

\footnotetext{
1*Lecturer, School of Psychology, Queen's University Belfast, University Road, Belfast, BT7 $1 \mathrm{NN} ;{ }^{2}$ Specialist Surgical Dentist, ${ }^{3}$ Consultant/Senior Lecturer in Oral Surgery, ${ }^{4}$ Consultant/ Senior Lecturer in Oral Surgery \& Director of Dental Education, School of Dentistry, Queen's University Belfast, University Road, Belfast, BT7 1NN

${ }^{*}$ Correspondence to: Dr Martin Dempster

Email:m.dempster@qub.ac.uk
}

\section{Refereed pape}

Accepted 7 November 2005

DOI: $10.1038 /$ sj.bdj.4814204

(c) British Dental Journal 2006; 201: 587-590
Research suggests that one of the instant dangers of binge drinking, especially for adolescents and young adults, is personal injury and vulnerability to physical assault, and that the 15 to 25 year age group is the modal group for alcohol-related facial trauma, assaults and for facial injuries occurring in public bars or on the street. ${ }^{4}$ Indeed, Shepherd and colleagues ${ }^{5}$ have shown that severity of facial injury can be related to the amount of alcohol consumed by the victim. Their data showed that abstinence made severe or multiple injuries less likely, and severity of injury increased as alcohol consumption increased. Heavy or 'binge' drinking, especially more than 10 units, conferred greatest risk of severe injury.

A review of research on drinking behaviour among young people states that binge drinking is not considered risky by young people and the adverse consequences normally cited (such as physiological effects) are seen to be acceptable. ${ }^{6}$ Long term effects such as liver damage have no immediacy for adolescents and they often perceive outcomes such as this to be something they can avoid in the longer term. Therefore, in order for interventions designed to reduce binge drinking among adolescents to be effective, they must encourage adolescents to realise that binge drinking has potentially serious immediate and long term consequences which are salient for adolescents. To date, no published research exists about the effect of such an intervention on this 'at-risk' group. Most previous research has relied on intervention after the 'event' at A \& E units. ${ }^{7,8}$

The aim of the present unique study was to evaluate the effectiveness of an orofacial trauma-based brief intervention, designed to raise adolescent males' awareness about the immediate dangers of binge drinking, and delivered in a classroom setting. It is possible that this could be adopted in the GDS context for the purposes of opportunistic primary intervention.

\section{METHOD}

\section{Construction of intervention}

The intervention consisted of a PowerPoint presentation and accompanying dialogue, delivered by JM and lasting for 20 min. It briefly summarised current loco-regional statistics, and included photographs of selected actual cases of individuals with facial trauma. It also highlighted the ways in which quality of life had been altered for the individuals concerned. 


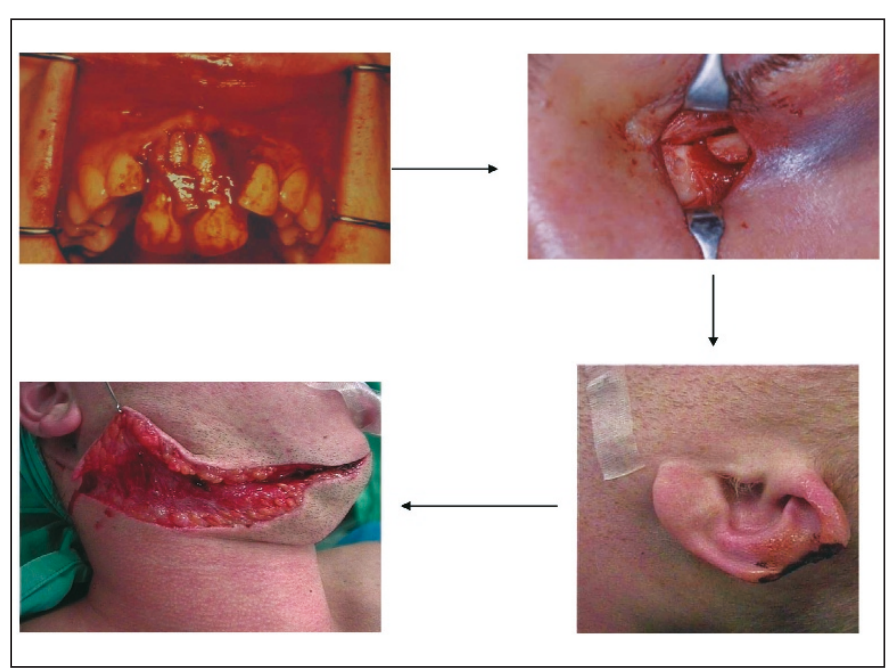

Fig. 1 An example of the order of some of the images used in the intervention illustrating increasing severity

\begin{tabular}{|l|l|l|}
\multicolumn{2}{|c}{ Table 1 Dropout rates for participants at each stage of the study } \\
\hline & Intervention Group & Control Group \\
\hline Baseline & $n=133$ & $n=49$ \\
\hline Post-presentation & $n=133$ & not applicable \\
\hline Follow-up & $n=121(91 \%)$ & $n=47(96 \%)$ \\
\hline
\end{tabular}

Photographs were accompanied by a brief narrative describing the circumstances of the injury and the age of the victim. Brief information was provided about how these injuries were treated including the long-term effects of such injuries, such as altered sensation or ability to chew, as well as obvious disfigurement.

Injuries shown were initially relatively minor, such as traumatized teeth, and then moved on to more serious injuries such as fractured facial bones. Finally, severe disfiguring injuries such as extensive facial lacerations were shown (Fig. 1).

In constructing the intervention, cognisance was taken of the potential 'active ingredients'. These were considered to be:

- The visual impact of increasingly adverse consequences

- The dialogue (delivered by the same presenter to limit bias), which illustrated the salient points of each case, especially the long term morbidity associated with the injuries sustained

- The fact that these were real cases.

The Theory of Planned Behaviour ${ }^{9}$ has been shown to be a useful explanatory model of binge drinking among young people. ${ }^{10,11}$ The presentation was designed to address the cognitive components of this model, which may in turn cause desirable behavioural changes. Specifically, the Theory of Planned Behaviour suggests several components which interact to determine behavioural intention. These components are:

- Beliefs about the outcomes of the behaviour and evaluations of these outcomes

- Beliefs about others' attitudes to the behaviour and motivation to comply with others

- The perceived control over the behaviour.

In short, the presentation attempted to communicate to the young people that the consequences of binge drinking can be serious and undesirable, that their peers disapprove of the violent behaviour that can result from binge drinking and that they have the ability to control this behaviour, thereby basing our message on the key elements of a popular behavioural model. The intention was not to shock, but to motivate through education and consequently modify cognitive processes about binge drinking.

\section{Pilot study}

School, parental and self consent was obtained from 60 male pupils in the pilot school. All pupils were aged 15 or 16 years old. Questionnaires were administered to the pupils in a classroom setting immediately before and following the intervention and at one month after the intervention. A total of 60 students completed the baseline questionnaires, 57 completed the postpresentation questionnaires and 55 completed the follow-up questionnaire.

The questionnaires addressed the adolescents' drinking behaviour as well as examining beliefs about the consequences of binge drinking, perceived norms and perceived control about the behaviour, and intention to binge drink. These latter four constructs are considered to be useful predictors of binge drinking behaviour among young people. ${ }^{10}$ The questionnaires used were based on the guidelines for developing questions using the Theory of Planned Behaviour. ${ }^{9}$ The actual questions have been used previously among university students and have been shown to be psychometrically sound. ${ }^{10,11}$

The pilot study was conducted in order to finalise the design and delivery of the intervention and questionnaires. Observer feedback was assessed at three time points during the presentation to gauge the effects of increasing severity of the injuries and feed back into the design. In addition, the pilot study results further informed the sample size calculation for the main study, which indicated that 165 participants would be sufficient to detect a difference (assuming an effect size of partial $\eta^{2}=0.06$ ) between the intervention group and the control group with 90\% power and an alpha value of 0.05 , using an analysis of covariance with baseline scores as the covariate and follow-up scores as the outcome.

\section{Definitive study (exploratory trial)}

School, parental and self consent was obtained from 182 male pupils in five Belfast schools. All schools were located in an urban area. Approximately 75\% of the control group and 76\% of the intervention group were obtained from grammar schools, with the remainder in each group obtained from secondary comprehensive schools. In Northern Ireland, admission to grammar schools is based on high attainment on achievement tests taken by pupils at age 11 years. Pupils who are not admitted to grammar schools attend secondary comprehensive schools. Ensuring that the control and intervention groups had approximately similar proportions of grammar school attendees was considered the most efficient method of ensuring that educational attainment and socio-economic status was similar in both groups. All pupils were aged 15 or 16 years old. Questionnaires were administered to the pupils in a classroom setting immediately before and following the intervention and at one month after the intervention with the exception of the control group, where the post-presentation questionnaire was omitted (Table 1).

Descriptive data regarding participants' drinking behaviour was calculated (using frequencies and percentages). Differences between the two groups in terms of the changes in attitudes and thoughts about binge drinking were analysed using analysis of covariance models (with baseline scores as the covariate and one month follow-up scores as the outcome variable).

\section{RESULTS}

Approximately $87 \%(143 / 165)$ of those who responded to the question reported that they had drunk alcohol at some time in their lives. At the time of the study, 87\% (124/143) of the participants who had previously drunk alcohol still drank alcohol (68\% of the entire sample). The majority of those who drank alcohol (78.4\%) reported obtaining their alcohol from an off-licence. In addition, a majority of drinkers (58.4\%) indicated that they often or sometimes obtained alcohol from a bar or club. Beer, lager or 
Table 2 Problems experienced as a result of being drunk (definitive study)

\begin{tabular}{|c|c|c|c|c|c|}
\hline & Frequency & $\begin{array}{l}\text { Percent of drinkers } \\
\qquad(n=143)\end{array}$ & $\begin{array}{l}\text { Percent of } \\
\text { intervention group } \\
(n=133)\end{array}$ & $\begin{array}{c}\text { Percent of control } \\
\text { group } \\
(n=49)\end{array}$ & $\begin{array}{l}\text { Percent of total } \\
\quad(n=182)\end{array}$ \\
\hline Damage to an object or clothing & 66 & 46.2 & 36.1 & 36.7 & 36.3 \\
\hline Quarrel or an argument & 53 & 37.1 & 32.3 & 20.4 & 29.1 \\
\hline Loss of money or other valuables & 46 & 32.2 & 27.1 & 20.4 & 25.3 \\
\hline Scuffle or fight & 38 & 26.6 & 25.6 & $8.2^{*}$ & 20.9 \\
\hline Accident or injury & 33 & 23.1 & 18.8 & 16.3 & 18.1 \\
\hline Problems in your relationship with your friends & 33 & 23.1 & 17.3 & 20.4 & 18.1 \\
\hline Problems in your relationship with your parents & 26 & 18.2 & 15.0 & 12.2 & 14.3 \\
\hline Trouble with police & 26 & 18.2 & 14.3 & 14.3 & 14.3 \\
\hline Poor performance in school & 13 & 9.1 & 7.5 & 6.1 & 7.1 \\
\hline Being the victim of robbery or theft & 6 & 4.2 & 4.5 & 0.0 & 3.3 \\
\hline Attending hospital & 6 & 4.2 & 3.0 & 4.1 & 3.3 \\
\hline Problems in your relationships with your teachers & 5 & 3.5 & 2.3 & 4.1 & 2.7 \\
\hline
\end{tabular}

stout were the most popular drinks of choice (among 79.6\% of drinkers) and ready-mixed drinks were also frequently consumed among $63.3 \%$ of drinkers.

Participants were asked to indicate whether they had personal experience with any of a list of possible adverse sequelae of drinking alcohol, as a result of their own drinking behaviour (see Table 2). In terms of violent behaviour, for example, $37.1 \%$ of drinkers had experience of a quarrel or argument which they related to alcohol consumption, and $26.6 \%$ had been involved in a physical fight. Just over 23\% had experience of some form of injury or accident and 18.2\% of drinkers reported being in trouble in some way with the police.

At baseline, $48 \%(88 / 182)$ of the sample $(71 \%$ of current drinkers) were classified as binge drinkers (48\% vs $49 \%$ in the intervention and control groups respectively). At the follow-up stage, approximately $17 \%(11 / 64)$ of the baseline binge drinkers in the intervention group were no longer binge drinking and approximately $6 \%(1 / 18)$ of the baseline binge drinkers from the control group were no longer binge drinking. Although the pattern is in the desired direction, the difference in the proportion of binge drinkers was not statistically significant $\left(\mathrm{X}^{2}=1.52\right.$, $p=0.217)$. There was also no statistically significant difference between the two groups in terms of the number of units of alcohol consumed in a single session at one month follow-up, with baseline consumption of alcohol considered as a covariate $[\mathrm{F}(1,87)=$ $1.58, \mathrm{p}=0.21$.

Of the original 88 binge drinkers at baseline, 14 (16\%) did not provide valid answers to all the remaining questions on the questionnaire at both points in time and so were excluded from further analyses.

Analysis of covariance (with baseline scores as the covariate) indicated that at one month follow-up, compared to the control group, the intervention group appraised binge drinking behaviour more negatively and believed more strongly that they could control their binge drinking behaviour. The intervention group also tended to show a stronger intention to stop binge drinking than the control group (see Table 3).

\section{DISCUSSION}

Among victims of assault, it has been found that $89 \%$ of fractures and $70 \%$ of other injuries affect the face or mouth in one district general hospital. ${ }^{12}$ The sequelae of assault may be physical (cosmetic, functional) and/or psychological (depression,
Table 3 Differences between intervention and control groups on intention to binge drink, behavioural beliefs, normative beliefs and control beliefs about binge drinking, at one month follow-up (with baseline scores as a covariate)

\begin{tabular}{|c|c|c|c|c|c|c|}
\hline & SS & df & MS & $\mathrm{F}$ & $\mathrm{p}$ & $\eta^{2}$ \\
\hline \multicolumn{7}{|c|}{ Intention to stop binge drinking: } \\
\hline Group & 4.042 & 1 & 4.042 & 3.087 & 0.083 & 0.042 \\
\hline Error & 92.939 & 71 & 1.309 & & & \\
\hline
\end{tabular}

\begin{tabular}{|c|c|c|c|c|c|c|}
\hline \multicolumn{7}{|c|}{ Behavioural beliefs (attitude to binge drinking): } \\
\hline Group & 21553.581 & 1 & 21553.581 & 28.645 & $<0.001$ & 0.287 \\
\hline Error & 53422.747 & 71 & 752.433 & & & \\
\hline
\end{tabular}

\begin{tabular}{|c|c|c|c|c|c|c|}
\hline \multicolumn{7}{|c|}{ Normative beliefs (perception of others' attitudes to binge drinking): } \\
\hline Group & 89.758 & 1 & 89.758 & 0.128 & 0.721 & 0.002 \\
\hline Error & 49631.935 & 71 & 699.041 & & & \\
\hline
\end{tabular}

\begin{tabular}{l|l|l|l|l|l|l|l|}
\hline \multicolumn{7}{|c|}{ Control beliefs (perception of self control over binge drinking): } \\
\hline Group & 2181.720 & 1 & 2181.720 & 12.604 & 0.001 & 0.151 \\
\hline Error & 12290.089 & 71 & 173.100 & & & \\
\end{tabular}

anxiety, post traumatic stress disorder). In one study, 23\% of young assault victims developed post traumatic stress disorder (PTSD). ${ }^{13}$

It is well known that there is a strong association between facial injury and alcohol consumption. This may reflect increased aggression by the assailant or the victim, or increased vulnerability to injury by the victim. ${ }^{14}$ Hutchison and colleagues ${ }^{4}$ demonstrated that $46 \%$ of people with facial injuries due to assault are aged between 15-25 years and 55\% of assaults are related to alcohol consumption, with $44 \%$ of people with facial fractures having consumed alcohol within four hours of their injury.

Against this background, a variety of approaches have been adopted to intervene and break the cycle of binge drinking. These include primary prevention through crime prevention partnerships; secondary prevention, through the use of 'teachable moment' brief interventions; and tertiary prevention - of PTSD. ${ }^{15}$ However, there 
has been no intervention targeted at the 'at risk' group of adolescents within the UK to date.

Adolescents would appear to be an ideal target group due to their recognised risk-taking behaviours associated with 'sensation-seeking', which peaks in adolescence and is commoner in males. ${ }^{16}$ Furthermore, behaviour initiated in adolescence may become 'habit' and continue into adulthood where habitual behaviour is very difficult to change. In addition, cognitive development has commenced, which allows increased capacity for hypothetical thinking, decision making and consideration of consequences. ${ }^{17}$

In the present study, where a 'brief interventional' format, linking alcohol consumption with interpersonal violence and resultant orofacial injury was used, the main health message was not that alcohol is 'wrong', but to highlight the dangers of alcohol misuse, specifically heavy single episode drinking ('binge drinking') among 15-16 year old male school students.

The results of the study suggest a very high rate of alcohol consumption among the sample, which concurs with figures found by Miller and Plant ${ }^{1}$ among adolescents in Northern Ireland, but is nonetheless worrying, with $68 \%$ being regular drinkers. Over $58 \%$ were able to obtain alcohol from licenced premises, which would appear to have major implications for the veracity of identity (and age) confirmation procedures at these venues. Forty-five percent of the drinkers drank outside. Whilst we have no evidence to support the assertion, it might be expected that such outdoor drinking may be more likely to predispose the drinkers to adverse consequences. Of particular note was the prevalence of sustained injury and hospital attendance for this group, as well as their contact with the police - all of which have implications for health care, law and order, and costs to society.

Although this drinking behaviour did not change significantly within the one month post-intervention, the intervention had a significantly positive effect on attitudes towards binge drinking and intention to stop binge drinking. In their 'readiness to change model', Prochaska and DiClemente ${ }^{18}$ describe the stages that an individual goes through in their attempt to change behaviour: precontemplation, contemplation, preparation, action and maintenance. Therefore it is not unexpected to observe an 'intention to change' without evidence of actual change in behaviour. The intervention would appear to have moved participants along the 'stages of change' and the authors' view is that this is a positive effect of the intervention which needs to be built upon. Motivational interviewing is an effective method of changing problem behaviours, including drinking behaviour, ${ }^{19,20}$ and this intervention may be seen as an important first stage in a motivational interviewing package - feedback of personal risk, enhancement of perceptions of personal control over drinking behaviour, and advice to change this behaviour. Other stages in motivational interviewing are: providing a menu of ways to change drinking behaviour, providing an empathetic counselling style, and improving the self-efficacy and optimism of the participants in relation to changing their drinking behaviour. ${ }^{21}$ These additional elements of the motivational interviewing approach could be incorporated as small group work and delivered in a classroom/tutorial setting, in an attempt to improve the overall effectiveness

The role of the GDP in this process is yet to be clarified. However, in much the same way as smoking cessation and other oral health promotion interventions have been delivered in an oppor- tunistic one-to-one fashion, it maybe that this user-friendly intervention might be adapted to allow intervention on a single patient basis. Practitioners may wish to have a pre-recorded CD version of this intervention for members of this 'at-risk' group in the waiting area and, where appropriate, provide supporting information at the time of their consultation. Nevertheless, further research is required before the results of this study could be generalised to settings outside the classroom.

Illustrating the connection between binge drinking and risk of orofacial trauma primarily affects attitudes to binge drinking among adolescents. Within this population it appears that attitude change is an important antecedent to allow interventions designed to reduce binge drinking to have their optimum effect. Therefore, the intervention tested here may be an appropriate precursor to a more in-depth psychosocial intervention, following the motivational interviewing approach.

\section{This research was supported by a grant from the British Academy.}

1. Miller P, Plant M. Drinking, smoking and illicit drug use among 15 and 16 year old school students in Northern Ireland. Northern Ireland: Department of Health, Social Services and Public Safety, 2001.

2. Health Promotion Agency Northern Ireland. Health behaviour of school children in Northern Ireland: a report on the 1997/1998 survey. Health Promotion Agency Northern Ireland, 2000.

3. The Licensing Act. London: Department for Culture, Media and Sport, 2003.

4. Hutchison I, Magennis P, Shepherd J, Brown A. The BAOMS United Kingdom survey of facial injuries part 1: aetiology and the association with alcohol consumption. Br J Oral Maxillofac Surg 1998; 36: 4-14.

5. Shepherd J, Irish M, Scully C, Leslie I. Alcohol intoxication and severity of injury in victims of assault. BrMed J 1988; 296: 1299.

6. Murgraff $V$, Parrott A, Bennett P. Risky single-occasion drinking amongst young people - definition, correlates, policy, and intervention: a broad overview of research findings. Alcohol Alcoholism 1999; 34: 3-14.

7. Smith A, Shepherd J, Hodgson R. Brief Interventions for patients with alcohol-related trauma. Br J Oral Maxillofac Surg 1998; 6: 408-415.

8. Monti P, Colby S, Barnett N et al. Brief intervention for harm reduction with alcohol positive older adolescents in a hospital emergency department. J Consult Clin Psychol 1999; 67: 989-994.

9. Ajzen I. The theory of planned behaviour. Organ Behav Hum Dec 1991; 50: 179-211.

10. Norman $P$, Bennett $P$, Lewis $H$. Understanding binge drinking among young people: an application of the theory of planned behaviour. Health Educ Res 1998; 13: 163-169.

11. Murgraff $V$, McDermott $M R$, Walsh J. Exploring attitude and belief correlates of adhering to the new guidelines for low-risk single-occasion drinking: an application of the theory of planned behaviour. Alcohol Alcoholism 2001; 36: 135-140.

12. Shepherd J, Scully C, Shapland M, Irish M, Leslie I. Assault: characteristics of victims attending an inner city hospital. Injury 1988; 19: 185-190.

13. Breslau N, Davis G, Andreski P, Peterson E. Traumatic events and post-traumatic stress disorder in an urban population of young adults. Arch Gen Psychiat 1991; 48: 216.

14. Magennis P, Shepherd J, Hutchison I, Brown A. Trends in facial injury. Br Med J 1998 316: $325-326$

15. Shepherd J, Qureshi R, Preston M, Levers B. Psychological distress after assaults and accidents. BrMed J 1990; 301:849-850

16. Greene K, Kremar M, Walters L, Rubin D, Hale L. Targeting adolescent risk-taking behaviors: the contributions of egocentrism and sensation-seeking. J Adolescence 2000; 23: 439-461.

17. Juszczak L, Sadler L. Adolescent development: setting the stage for influencing health behaviors. Adolescent Med 1999; 10: 1-11.

18. Prochaska J, DiClemente C. The transtheoretical approach: crossing the traditional boundaries of therapy. Illinois: Dorsey/Dow, Jones-Irwin, 1984.

19. Ritson B. Treatment for alcohol related problems. Br Med J 2005; 330: 139-141.

20. World Health Organization (WHO) Brief Intervention Study Group. A cross-national trial of brief interventions with heavy drinkers. Am J Public Health 1996; 86: 948-955.

21. Miller W R, Sanchez V C. Motivating young adults for treatment and lifestyle change. In Howard G (ed) Issues in alcohol use and misuse in young adults. Notre Dame: University of Notre Dame Press, 1993. 